\title{
Wavelet analysis in a structured clay soil using 2-D images
}

\author{
J. A. Piñuela ${ }^{1}$, D. Andina ${ }^{2}$, K. J. McInnes ${ }^{3}$, and A. M. Tarquis ${ }^{4}$ \\ ${ }^{1}$ Universidad Europea de Madrid, Villaviciosa de Odón, Madrid, 28040, Spain \\ ${ }^{2}$ Dpto. de Señales, Sistemas y Radiocomunicación. E.T.S. Ingenieros de Telecomunicaciones, U.P.M. Ciudad Universitaria \\ s.n. Madrid 28040, Spain \\ ${ }^{3}$ Dept. of Soil and Crop Sciences. Texas A\&M University, 2474 Tamu, College Station, TX 77843, USA \\ ${ }^{4}$ Dpto. de Matemática Aplicada. E.T.S. Ingenieros Agrónomos, U.P.M. Ciudad Universitaria s.n. Madrid 28040, Spain
}

Received: 18 December 2006 - Revised: 15 June 2007 - Accepted: 15 June 2007 - Published: 20 July 2007

\begin{abstract}
The spatial variability of preferential pathways for water and chemical transport in a field soil, as visualized through dye infiltration experiments, was studied by applying multifractal and wavelet transform analysis (WTA). After dye infiltration into a $4 \mathrm{~m}^{2}$ plot located on a Vertisol soil near College Station, Texas, horizontal planes in the subsoil were exposed at $5 \mathrm{~cm}$ intervals, and dye stain patterns were photographed. Box-counting methods and WTA were applied to all of the 16 digitalized high-resolution dye images and to the dye-mass image obtained merging all sections. The wellknown Devil's staircase multifractal was also used to illustrate wavelet-based analysis. Our results suggest that wavelet methods can complement box-counting analysis in the context of multiscaling structure analysis.
\end{abstract}

\section{Introduction}

The study of water movement and transport of chemicals in soils is of fundamental importance in hydrologic science. It is generally accepted that in most soils, water and solutes may flow through preferential paths. The spatial variability of these preferential pathways in a field soil, as visualized through dye infiltration experiments are of special importance in determining if and how fast contaminants reach ground water (Tarquis et al., 2006).

A common approach to describe dye patterns has been by descriptive statistics of the vertical variation in dye coverage as estimated from high-resolution dye images taken at varying depth increments. Frequently, these images show a complex pattern that are unpredictable in detail, but predictable in the sense that smaller pieces of the pattern, when suitably enlarged, are statistically similar to larger pieces of the pattern.

Correspondence to: J. A. Piñuela

(juan.pinuela@uem.es)
This property of statistical self-similarity can be quantified using fractal geometry (Pachepsky et al., 2000).

Previous work done by some of the authors (Tarquis et al., 2006) describes the scaling/multiscaling behavior of dyestained flow paths calculating the maximum configuration entropy $(H(L))$, the characteristic length $(L)$ and the generalized dimensions $\left(D_{q}\right)$. The work presented herein focuses on multifractal spectra and includes computation of the multifractal spectrum $f(\alpha)$. The multifractal spectrum $f(\alpha)$ provides a detailed distribution of the singularities of the signal and can be considered more general than generalized dimensions $D_{q}$. The relation between these two parameters is established through the mass exponent $\tau_{q}$ as:

$D_{q}=\frac{\tau_{q}}{q-1}$

$f(\alpha)=q \alpha(q)-\tau_{q}$

However, other parameters such as configuration entropy have shown their relevance and are also of great importance while characterizing flow paths. For this reason these parameters are briefly described in Sect. 2 .

With respect to multifractal related parameters, the application of the wavelet transform modulus maxima (WTMM) representation of a signal has almost reached the status of a standard. The wavelet related multifractal formalism was first developed in Arneodo et al. (1988) and has been extensively used to test many natural phenomena and has contributed to substantial progress in each domain in which it has been applied (Struzik, 1999). However, in the case of multidimensional signals such as images, there are ambiguities (Hsung et al., 1999) in tracing the maxima curves of the WTMM, so new methods are needed (Zhong and Ning, 2005). Thus, the objectives of this work were to describe how wavelet theory can be used to solve multifractal analysis (global estimates) and compare the method with boxcounting-based analysis.

Published by Copernicus Publications on behalf of the European Geosciences Union and the American Geophysical Union. 

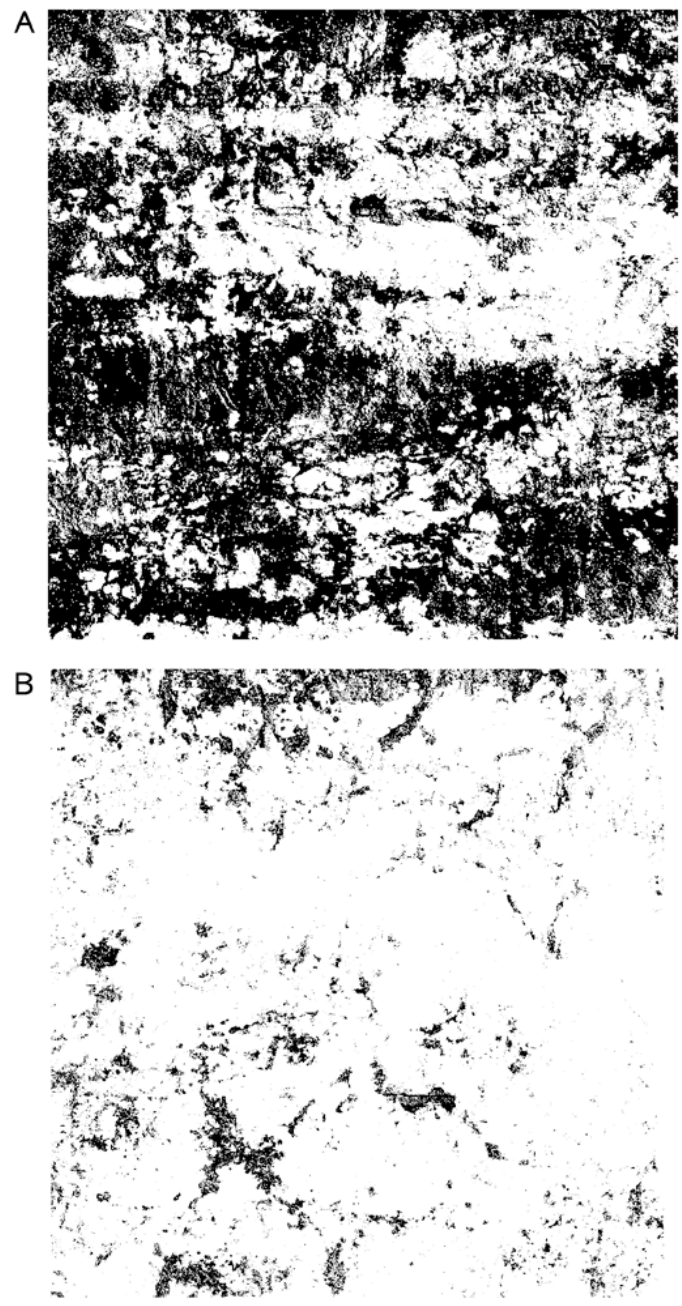

Fig. 1. Images of horizontal sections of $2 \times 2 \mathrm{~m}$ corresponding to a depth of: (A) $20 \mathrm{~cm}$ and (B) $30 \mathrm{com}$. Black pixels represent dye stained areas.

The organization of the paper is as follows. First other parameters such as configuration entropy are revisited. Next, a necessary introduction to wavelet transform and singularity measures is given as well as a definition of the socalled Wavelet Transform Modulus Maxima (WTMM) (Mallat, 1999). The extension to multifractal one dimensional signals is covered next and experiments on soil images are described. Finally some conclusions and future work research directions are given.

\section{Methodology and previous results}

\subsection{Dye tracer experiment}

The experiments were conducted in a five hectare agricultural research site located on the Brazos River floodplain near College Station, Texas. The field in which the plot was lo- cated is mainly used to grow cotton, corn, grain sorghum, and small grain, and improved pasture. At the time of the experiment the soil was tilled after a corn crop and a plot of $2 \times 2 \mathrm{~m}$ was selected for this study.

The selected plot was irrigated for a few days to establish a uniform and steady moisture level throughout the profile prior to the experiment. After wetting, irrigation water with $30 \mathrm{~g} / 1$ brilliant blue dye (FCF) was applied uniformly with an automated spray system. About six days after the irrigation with the dye, parallel horizontal sections spaced $5 \mathrm{~cm}$ apart were successively excavated until no dye stained soil was found, resulting in 15 sections. A $35-\mathrm{mm}$ camera with Kodachrome 60 film was used to photograph each of the horizontal sections as they emerged during the excavation. Sixteen sections were photographed: the surface and fifteen subsurface sections.

The $2 \times 2 \mathrm{~m}$ horizontal section at any given depth was represented by a matrix of $2048 \times 2048$ pixels. Each pixel represented an area of approximately $1 \mathrm{~mm}^{2}$. The value of each pixel was either black (dye stained) or white (unstained), so we had two-dimensional binary images like the ones shown in Fig. 1.

\subsection{Configuration entropy}

Configuration entropy analysis studies the effect of scale in any measure (a scalar quantity that leads to a positive distribution) defined in a plane. If a plane were divided into an arbitrary number of smaller areas (e.g., boxes) and the measure $(\mu)$ were estimated in each sub-area, a distribution of the measure would be obtained. Estimation of dye patterns from a binary image implies counting pixels representing dyed areas and expressing this count as a percentage of the total number of pixels in the image. We refer to this quantity as Dye Tracer percentage (DT) and it is the basis of the configuration entropy.

Mathematically, the probability associated with a case of $j$ dye pixels in a box of size $r$ is defined as:

$p_{j}(r)=\frac{N_{j}(r)}{n(r)}$.

where $N_{j}(r)$ is the number of boxes with $j$ dye pixels and $n(r)$ is the number of boxes of size $r$. The configuration entropy is thus defined as:

$H(r)=-\sum_{j=0}^{r \times r} p_{j}(r) \log \left(p_{j}(r)\right)$.

$H(r)$ as expresses in Eq. (4) reveals the uncertainty associated with the DT. For proper comparison $H(r)$ needs to be normalized resulting in:

$H^{*}(r)=\frac{H(r)}{H_{\max }(r)}$ 


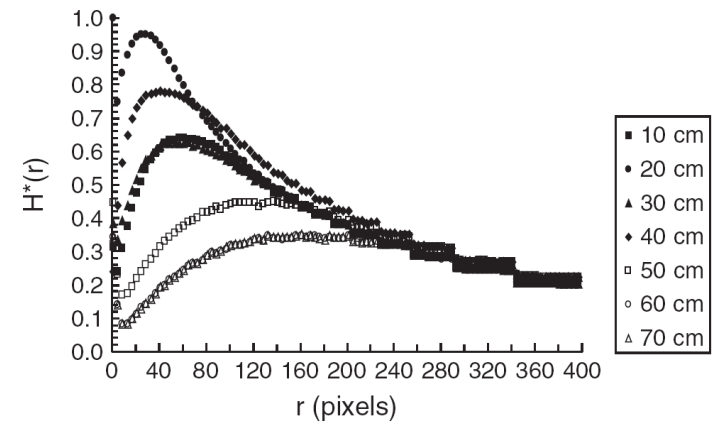

Fig. 2. Normalized configuration entropy $H^{*}(r)$ versus box size $r$ from horizontal sections at different depths.

where $H_{\max }(r)=\log \left(r^{2}+1\right)$. Plotting the normalized entropy versus $r$ could be used as a descriptor of the image morphology. In our experiment, for example, all the horizontal sections showed the same behavior in the configuration entropy curve (Fig. 2). The general pattern was a rapid increase in $H$ with $r$ followed by a gradual decay.

The first point of the curve, for $r=1$, was totally correlated with the DT of the horizontal section. Note that the decrease in DT with depth was not linear, but showed abrupt changes. By a depth of $15 \mathrm{~cm}$, the DT value had decreased to half of that at the surface, and by $30 \mathrm{~cm}$ depth the value was $10 \%$.

\section{Wavelets and singularities}

\subsection{Introduction}

Wavelet theory has its origin in several disciplines. The types of functions that are now called wavelets were studied in quantum field theory, signal analysis, and function space theory. In all these areas, wavelet-like algorithms replaced the classical "windowed" Fourier transform.

The windowed Fourier transform serves as means to describe or compare the fine structure of a function at different resolutions. Its basic building blocks are the integer dilates of the sine and cosine functions multiplied by a "window" function. Although quite successful, this method is not applicable to highly localized structures when the window size is fixed.

To overcome these problems, one replaces sine and cosine by a function that has compact support and its dilates and translates form an orthonormal basis of the function space being considered (usually $L^{2}(\mathbb{R})$ ). The famous Daubechies wavelets (Daubechies, 1992) are an example of these wavelet bases. Other examples, like the derivative of a Gaussian that will be used in our work are also sketched in (Fig. 3)

It can be shown that under certain conditions this type of function performs a multiresolution analysis or decomposition of L2(R). Such wavelet decompositions are obtained via a multiresolution analysis (Mallat, 1989). Therefore, the
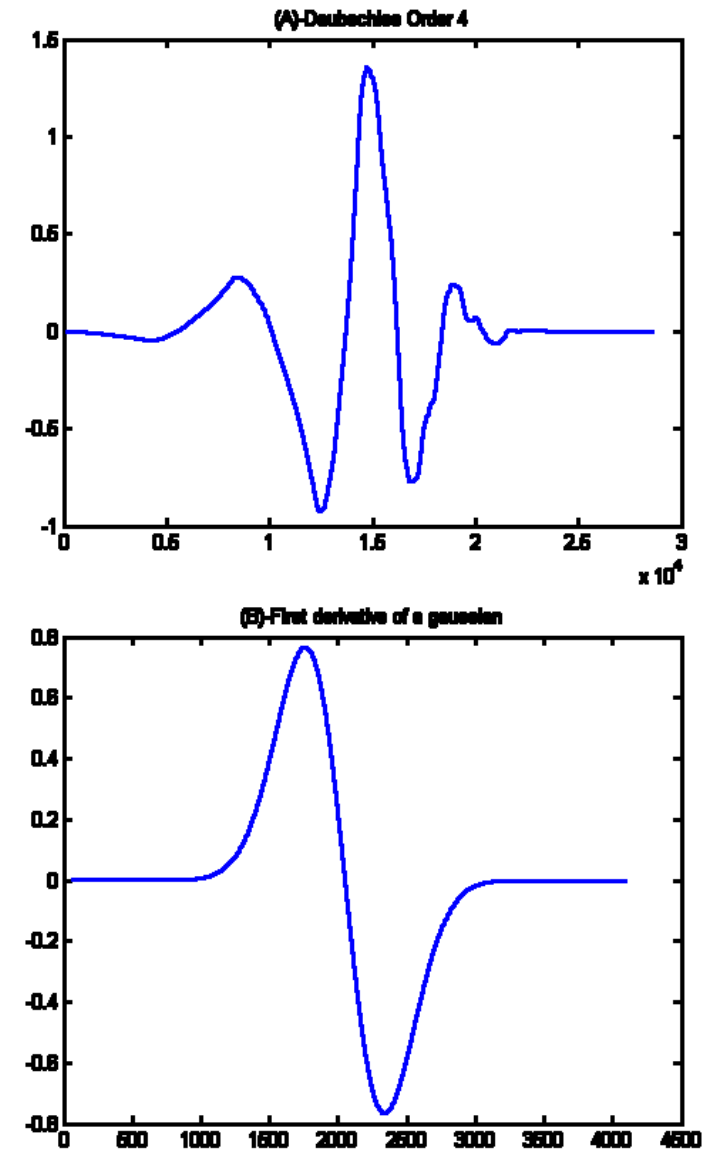

Fig. 3. Some examples of Wavelet bases: Daubechies of order 4, first derivative of a gaussian and Mexican Hat wavelets.

main feature we are interested in, is the ability of wavelet transform to focus on localized signal structures performing a multiscale (multiresolution) analysis of signal singularities. Next, we extend this type of analysis to complex signals such as multifractals.

\subsection{Wavelet transform and Lipschitz regularity}

Conceptually, the continuous wavelet transform (CWT) is a convolution product of a signal with a scaled and translated kernel (usually a n-th derivative of a smoothing kernel in order to precisely detect singularities as pointed out later)

$$
W f(u, s)=\frac{1}{s} \int_{-\infty}^{\infty} f(x) \psi\left(\frac{x-u}{s}\right)
$$

Where $s$ and $u$ are real numbers $(s>0)$ which are discretized for computation purposes. In this way, the wavelet transform performs a transformation of a function $f(x)$ into a function defined over the scale-space plane (pair of values $u$ and $s$ ). As shown later, this transformation reveals more and more detail when observing smaller and smaller scales where the location of singularities can be detected. 

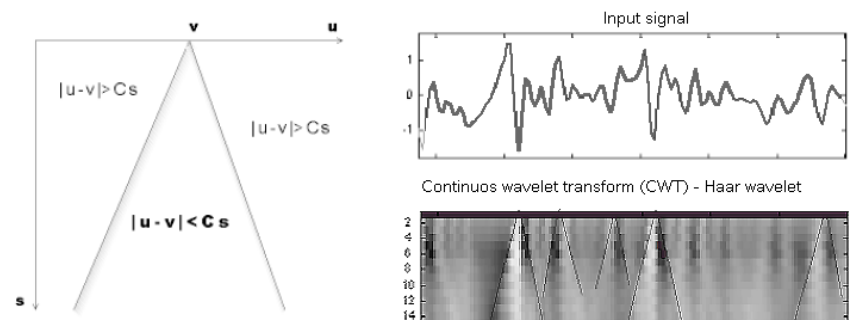

Continuos wavelet transform (CWT) - Haar wavelet

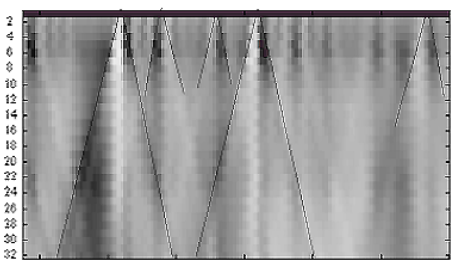

Fig. 4. Cone of influence of and abscissa $v$ and example.

To characterize singular structures, Lipschitz exponents are commonly used. They provide uniform regularity measurements over time intervals and local measures at any point. If $f$ has a singularity at $v$, which means that it is not differentiable at this point, the Lipschitz exponent at $v$ characterizes this singular behaviour.

Proper definitions of Lipschitz exponents are given next:

- A function $f$ is pointwise Lipschitz $\alpha \geq 0$ at $v$ if there exist $K>0$ and a polynomial $p_{v}$ of degree $m=\lfloor\alpha\rfloor$ such that:

$$
\forall t \in \mathbb{R},\left|f(t)-p_{v}(t)\right| \leq k|t-v|^{\alpha}
$$

- A function $f$ is uniformly Lipschitz $\alpha$ over $[a, b]$ if it satisfies (7) for all $v \in[a, b]$, with a constant $K$ independent of $v$.

To measure the local regularity of a signal is crucial to choose a wavelet with enough vanishing moments. A wavelet function $\psi(t)$ is said to have $n>\alpha$ vanishing moments if and only if:

$$
\int_{-\infty}^{\infty} t^{k} \psi(t) d t=0 \quad \text { for } \quad 0 \leq k \leq n
$$

It can be shown that a wavelet with $n$ vanishing moments can be written as the $n^{\text {th }}$ order derivative of a function $\theta$, so the resulting wavelet transform is a multiscale differential operator which is able to detect and isolate singularities up to exponents $\alpha \leq n$, such as the following theorem from establishes.

Theorem (Mallat, 1999)

If $f \in L^{2}(\mathbb{R})$ is uniformly Lipschitz $\alpha \leq n$ over $[a, b]$ then there exists $A>0$ such that:

$\forall(u, s) \in[a, b] x \mathbb{R} \quad(s>0) \quad \mid W f(u, s) \leq A s^{\alpha+\frac{1}{2}}$

Conversely, if $W f(u, s)$ satisfies Eq. (9) and if $\alpha<n$ is not an integer then $f$ is uniformly Lipschitz $\alpha$ on $[a+\epsilon, b-\epsilon]$ for any $\epsilon>0$.
When studying the regularity at point $v$ for one dimensional signals, we have to consider only those points $u$ which are in the cone of influence of $v$ in the scale-space plane defined by the CTW. The Fig. 4 shows the definition of the cone of influence of the singularity $v$ at the left side while an example for a signal is given to the right where the cones of influences of several singularities are highlighted.

In Fig. 4 we shall suppose that the analyzing wavelet has a compact support equal to $[-C, C]$, so the support of a dilated and translated version such as $\psi\left(\frac{t-u}{s}\right)$ is equal to $[u-C s, u+C s]$ and this is the cone of influence of $v$. In this way, we have to analyze the wavelet transform values inside these cones so that Eq. (9) remains valid as a necessary and sufficient condition for pointwise regularity computation.

As a concluding remark of this section, it must be pointed out that a wavelet able to detect any singularity such as the derivatives of the Gaussian always will be a worse choice than a wavelet fitting the actual range of singularities because the more vanishing moments produce longer effective supports and, as a consequence, coarser estimations.

\subsection{Wavelet transform modulus maxima (WTMM)}

We use the term modulus maximum (strict maximum) to describe any point $\left(u_{0}, s_{0}\right)$ such that $\left|W f\left(u, s_{0}\right)\right|$ is locally maximum at $u=u_{0}$. We call maxima line to any connected curve $s(u)$ in the scale-space plane $(u, s)$ along which all points are modulus maxima.

It can be shown (Mallat, 1999) that:

- Singularities can be detected finding the abscissa where the wavelet modulus maxima converge at fine scales.

- Pointwise regularity $\left(\alpha+\frac{1}{2}\right)$ can be calculated by measuring the decay slope of $\log _{2}|W f(u, s)|$ as a function of $\log _{2}(s)$ at the finest scales. So measuring the decay in the time-scale plane as suggested in Eq. (9) is not necessary. We can control it from its local maxima values connected via the maxima lines.

The Fig. 5 shows a signal with a sharp transition at and its corresponding continuous wavelet transform. We compute the WTMM (Wavelet transform modulus maxima) and store values of the modulus in the maxima lines that converge to the singularity. The decay of the modulus along these maxima lines are given to the right for the ridges numbered as 9,7 and 4 (plotted using continuous, dashed and dotted lines respectively). A simple linear regression may be used in order to compute the desired Lipschitz exponents.

\subsection{Extension to images}

For illustration of the extension to images we consider a path of $512 \times 512$ pixels of the dye mass image used in our experiments, which reflects the amount of dye stained pixels under the point being considered as shown in Fig. 7 (darker areas corresponds to higher values). 


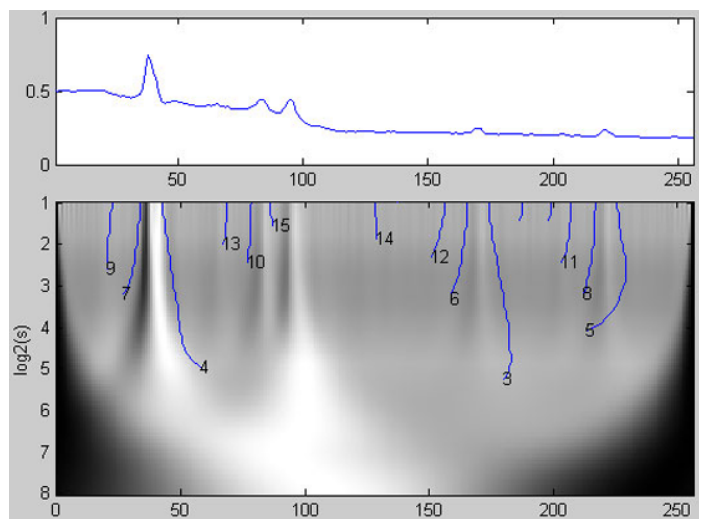

Fig. 5. Wavelet Transform Modulus Maxima and maxima chains.

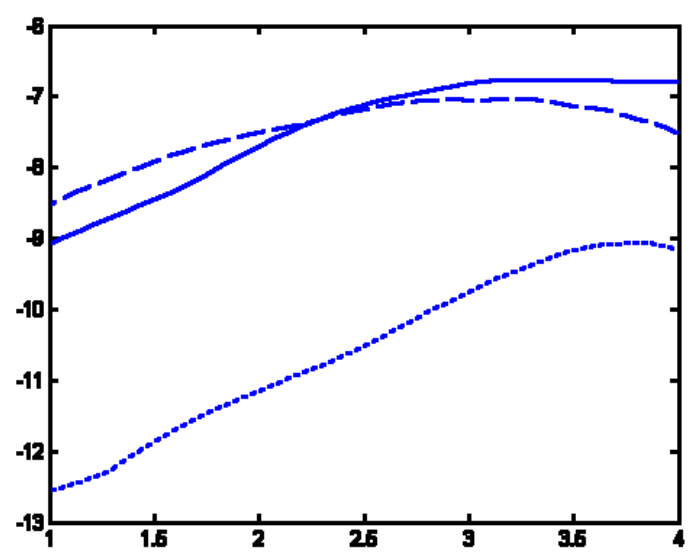

Fig. 6. Decay of modulus amplitude as function of scale gives Lipschitz exponents.

The extension of the previous concepts to images, or multidimensional signals in general, is conceptually simple, but cumbersome. For the two dimensional case the modulus of the wavelet transform is given by:

$M f(u, v, s)=\sqrt{\left|W^{1} f(u, v, s)\right|^{2}+\left|W^{2} f(u, v, s)\right|^{2}}$

with $u$ and $v$ denoting the two dimensional coordinates and the scale parameter being usually used as $s=2^{j}$. Now, we compute two separates wavelet transforms: $W^{1}$ refers to the wavelet transform performed along the horizontal dimension and $W^{2}$ refers to the vertical one.

Apart from the modulus, information about the angle is required in order to detect modulus maxima points which are defined as local maxima along the gradient direction which are initially expressed as:

$A f(u, v, s)=\tan ^{-1}\left(\frac{W^{2} f(u, v, s)}{W^{1} f(u, v, s)}\right)$

Next, we show different examples of the results obtained. The CWT has been computed for 20 consecutive scales along

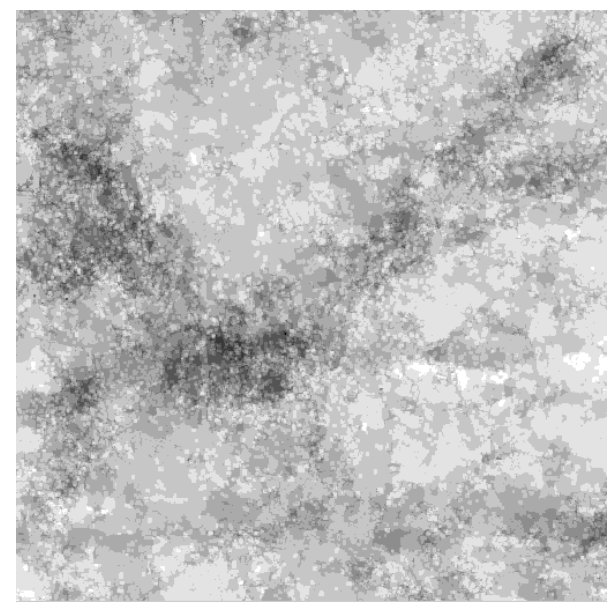

Fig. 7. A $512 \times 512$ path of the dye image of the experiment.

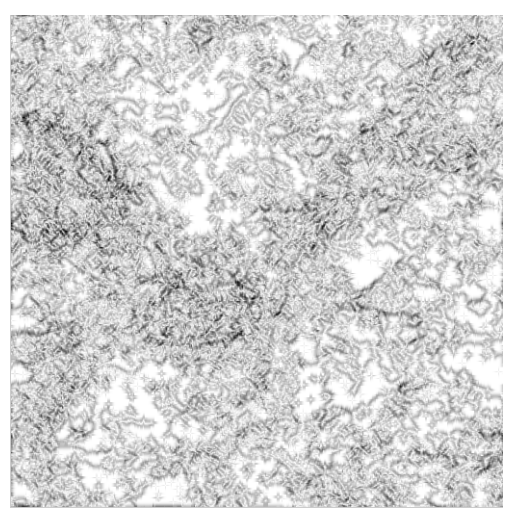

Fig. 8. Modulus of the CWT at scale $s=2$.

vertical and horizontal dimensions. The modulus of these images are shown in Fig. 8 for $s=2$ and in Fig. 9 for $s=8$. If we extract maxima values we have images like the ones showed in Fig. 10 and in Fig. 11.

\section{Wavelets and multifractal analysis}

\subsection{Multifractal formalism}

By multifractal structure we mean that there exists a particular arrangement of the points in an image in the so-called fractal components. Those fractals components are sets defined by the property that the image undergoes the same kind of change (transition or singularity) for all the points in the same component.

This multifractal formalism has two advantages. First, the multifractal structure leads to well defined statistical properties and secondly the fractal components are of great geometrical relevance. But for being able to apply the multifractal formalism it is necessary to develop techniques for the correct decomposition of images into their fractal components. 


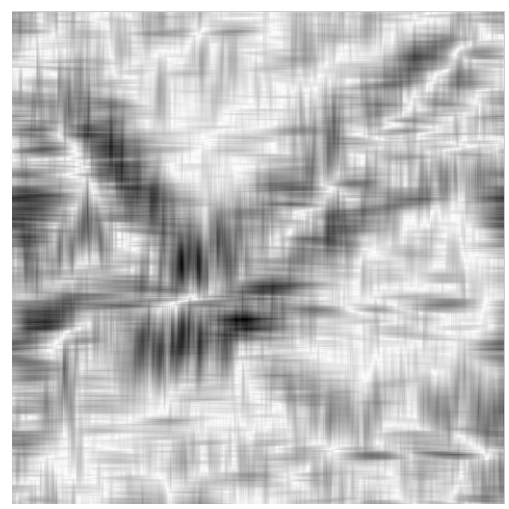

Fig. 9. Modulus of the CWT at scale $s=8$.

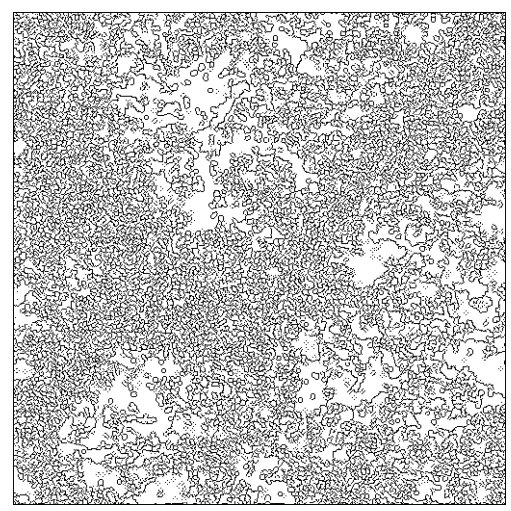

Fig. 10. Modulus maxima of the CWT at scale $s=2$.

That decomposition can be done by means of the wavelet analysis.

First, for each pixel $x\left(u_{0}, v_{0}\right)$ its singularity exponent $\alpha$ is computed. Then, all the points in the image are arranged according to the value of their singularity exponent arriving at the well known multifractal spectrum $f(\alpha)$.

\subsection{Multifractal spectrum and wavelets}

The goal of a multifractal analysis must then be to estimate the singularity distribution. In this context the so called multifractal spectrum and its fractal dimension is used. With the help of the well-known Devil's staircase fractal we will show how to compute its multifractal spectrum through the wavelet transform.

A Devil's staircase is the integral of a Cantor measure whose recursive construction implies that the Devil's staircase is a self-similar function. Figure 12 displays the devil's staircase obtained with $p_{1}=0.475$ and $p_{2}=0.525$, its wavelet transform computed using the first derivative of a Gaussian and the modulus maxima lines.

Let $u_{n}(s)$ the position of all local maxima of the wavelet modulus transform. The partition function $\mathrm{Z}$ measures the

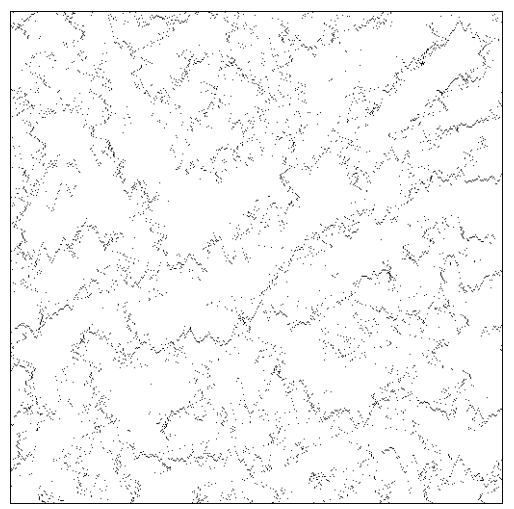

Fig. 11. Modulus maxima of the CWT at scale $s=8$.
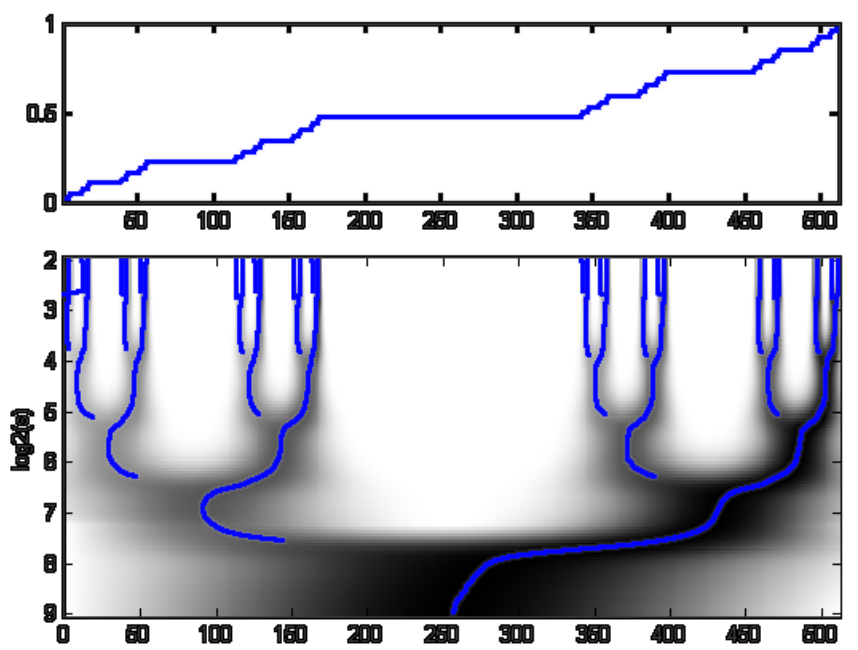

Fig. 12. Devil's staircase, wavelet transform and modulus maxima.

sum at power q of all these wavelet modulus maxima values:

$Z(q, s)=\sum_{n}\left|W f\left(u_{n}, s\right)\right|^{q}$

It is important to note that at each scale if there exist more than a maximum in the cone of influence, the sum includes only the maxima of largest amplitude.

For each $q$ (which is a real number) the scaling exponent measures the asymptotic decay of the partition function $Z(q, s)$ at fine scales:

$\tau_{q}=\lim _{s \rightarrow 0} \frac{\log Z(q, s)}{\log s}$

This means that $Z(q, s) \propto s^{\tau_{q}}$ and intuitively, since $q$ has the ability to select a desired range of values: small for $q<0$ and large for $q>0$, the scaling function globally captures the distribution of the Lipschitz exponents. Weak exponents are addressed with large negative $\mathrm{q}$, while strong exponents are suppressed. For large positive $q$, the converse takes place. 


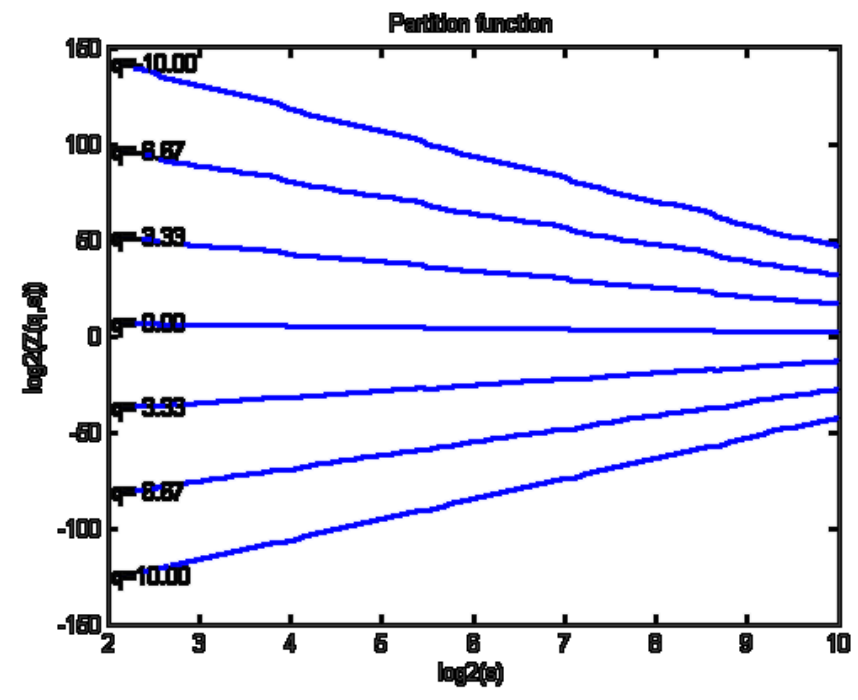

Fig. 13. Partition function for several values of $q$.

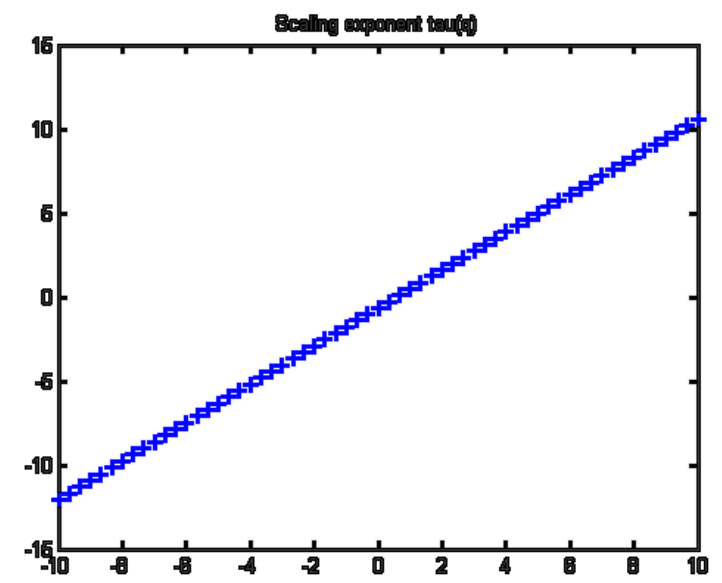

Fig. 14. Moments generating function.

Finally, using the inverse Legendre transform (which is applicable if and only if $f(\alpha)$ is convex) we obtain the multifractal spectrum $f(\alpha)$ as:

$f(\alpha)=\min _{q \in \mathbb{R}}\left(q\left(\alpha+\frac{1}{2}\right)-\tau(q)\right)$

where $f(\alpha)$ is convex when the signal is self-similar (colloquially speaking a measure is multifractals when its multifractal spectrum exists and has the shape of an inverted parabola). The spectrum $f(\alpha)$ reveals the distribution of singularities in a multifractal multifractal signal which is crucial to analyze its properties. The spectrum measures the global repartition of singularities having different Lipschitz regularity. For example, if the signal being considered were monofractal (only one component), the spectrum would consist of a single point. In the case of a multifractal as the devil's staircase or the dye stained images we are working

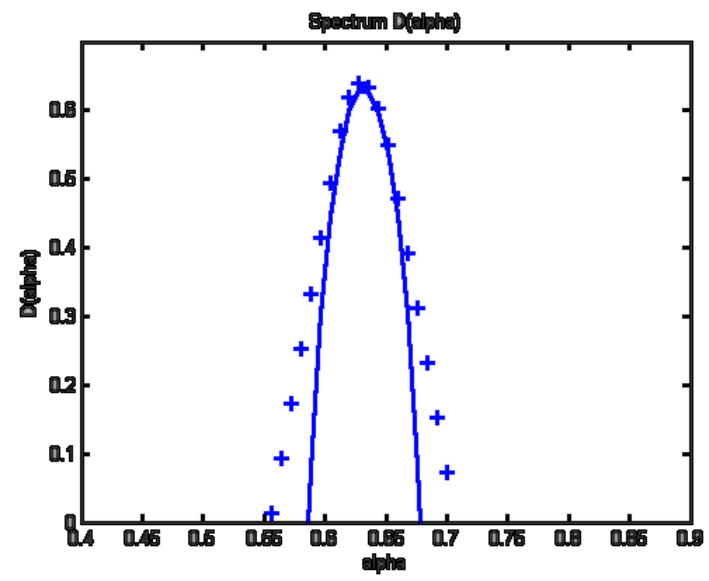

Fig. 15. $f(\alpha)$ values calculated with Eq. (14) and theoretical spectrum.

on, the spectrum range of $\alpha$-values increases according to the increase in the distribution heterogeneity. In conclusion, wider concave spectrum means more heterogeneity.

All these steps applied to the Devil's staircase example are shown in Fig. 13 where $Z(q, s)$ are plotted against scale for different $q$ values. Figure 14 which show $\tau_{q}$ and finally in Fig. 15 where values calculated with Eq. (14) and theoretical spectrum (Mallat, 1999) are compared.

The $f(\alpha)$ spectrum is related to the other commonly used set of multifractal exponents known as generalized fractal dimensions, calculated from the mass exponent function as:

$D_{q}=\frac{\tau_{q}}{q-1}$

The fractal dimension at $q=0$, equals the geometric support of the measure being studied (equals 1.0 for one dimensional signals or 2.0 for images). The information fractal dimension $D_{1}$ is obtained at $q=1$ using L'Hopital rule. A value of $D_{1}$ close to 1.0 characterize a system uniformly distributed throughout all scales, whereas $D_{1}$ close to 0 reflects a subset of the scale in which the irregularities are concentrated. With respect to $D_{2}$, simply said that is mathematically associated to the correlation function, so it measures the self-similarity of a signal.

For the Devil's example we have $D_{1}=0.6407$ which is near the theoretical value of $D_{1}=\frac{\log 2}{\log 3}=0.6309$. One of the reasons for the systematic difference between the theoretical and the computed multifractal spectrum might be in the computation of $Z(q, s)=\sum_{n}\left|W f\left(u_{n}, s\right)\right|^{q}$ where at some scales we may have an indeterminate function for $q<0$.

\section{Multifractal and wavelet based analysis of soil spatial variability}

Initially, most fractal theory applications in soil science use a monofractal approach, which assumes that soil spatial 


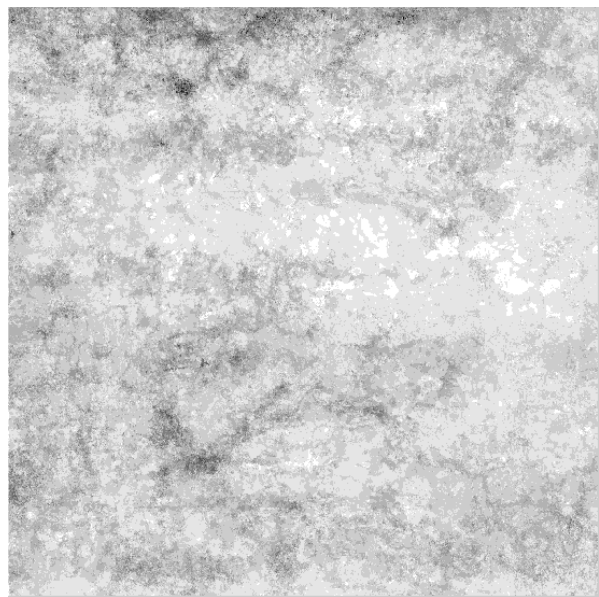

Fig. 16. Dye mass image being processed.

distribution can be uniquely characterized by a single fractal dimension (Kravchenko et al., 1999). However, a single fractal dimension might not always be sufficient to represent complex and heterogeneous behaviour of soil spatial variations.

Motivated by this, the work presented in Folorunso et al. (1994) found multifractal parameters to be superior to a single fractal dimension in distinguishing between soil types. Later Muller (1996) used multifractal analysis to characterize pore space in chalk and noticed that multifractal properties are closely related to chalk permeability and porosity.

For our application there are two types of experiments. First of all, horizontal sections, such as those of Fig. 1 may be analyzed separately looking for any scaling pattern. Finally the data from all 16 sections were merged to produce a spatial field of two measures: quantity of dye tracer (dye mass) and maximum dye infiltration depth (dye depth). The dye mass image is shown in Fig. 16 where darker values represent higher dye mass. Although, initially dye mass and dye depth quantities are integers in the range 0 to 16 (the same value as number of sections) proper normalization is needed in order to accurately compute power-law relationships between quantities and box size. For this reason the sum of all values is equalled to 1 prior to any computation.

\subsection{Box-counting methods for multifractal analysis}

A detailed description of the box-counting algorithm applied to our case study can be found in Tarquis et al. (2006), so only some results are given in order to analyze how wavelet analysis can complement box-counting algorithms. Figure 17 shows the resulting bi-log plots of the partition function versus box-size for different horizontal planes (only those corresponding to $20 \mathrm{~cm}$ and $30 \mathrm{~cm}$ are given). Partition function and generalized dimension for different values of $q$ are given in Figs. 18 and 19 for the dye-mass image.

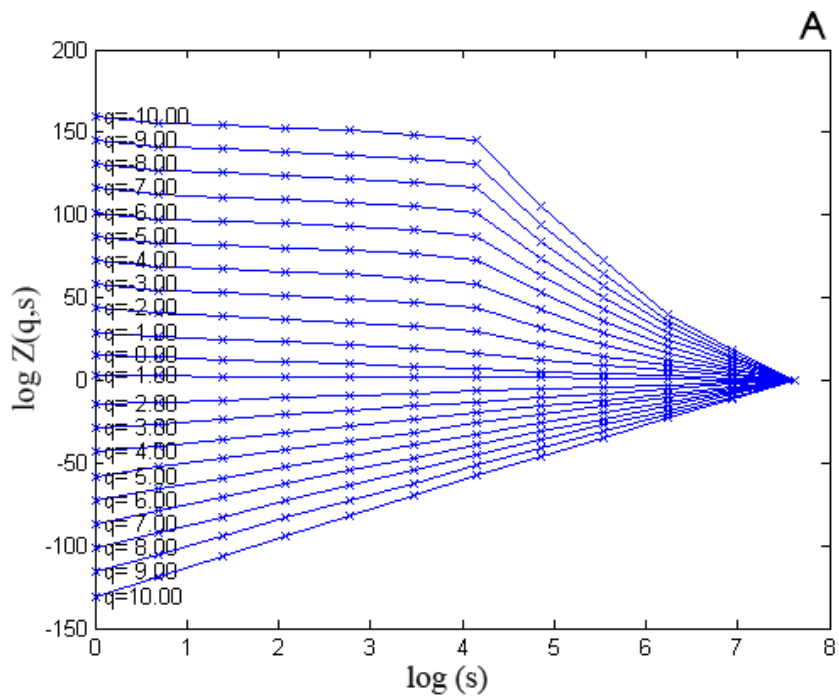

B

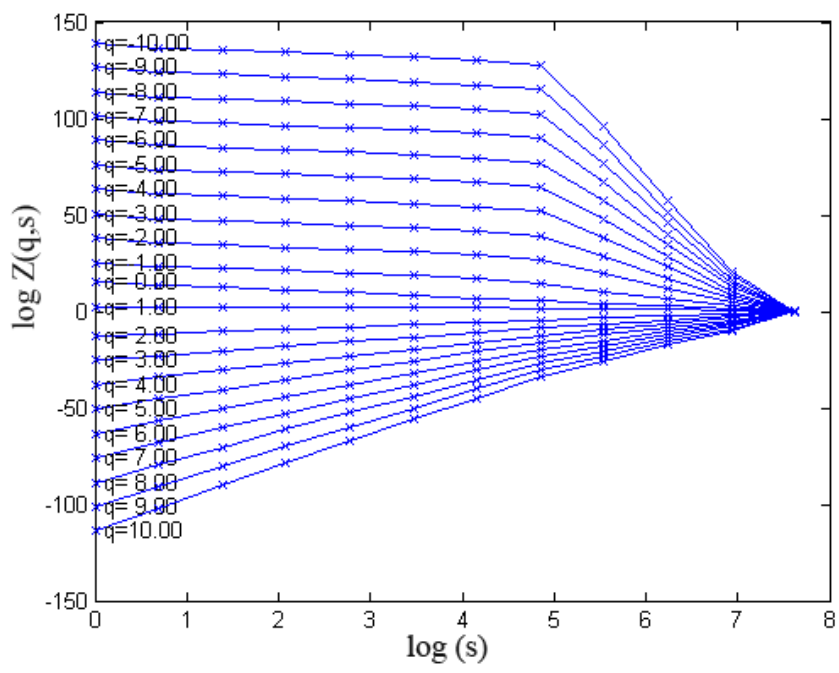

Fig. 17. Bi-log plot of partition function versus box-size for different sections: (A) $20 \mathrm{~cm}$ and (B) $30 \mathrm{~cm}$.

All partition functions showed a clear pattern in the data with two distinctive areas. One where there was a linear relationship and another where the slope was almost constant. So, only when the box-size passed a certain value a scaling pattern begins.

\subsection{Wavelet analysys (WTA)}

First experiments with the extension of methods developed in Sect. 4 to our case study showed an unstable behavior which are far from being expected. As mentioned before and pointed out by Hsung et al. (1999) and Zhong and Ning (2005) there are some ambiguities in tracing the maxima curves in scale planes when dealing with multidimensional signals. Apart from that, the most important result is that, as box-counting analysis reveals that multifractal behavior 


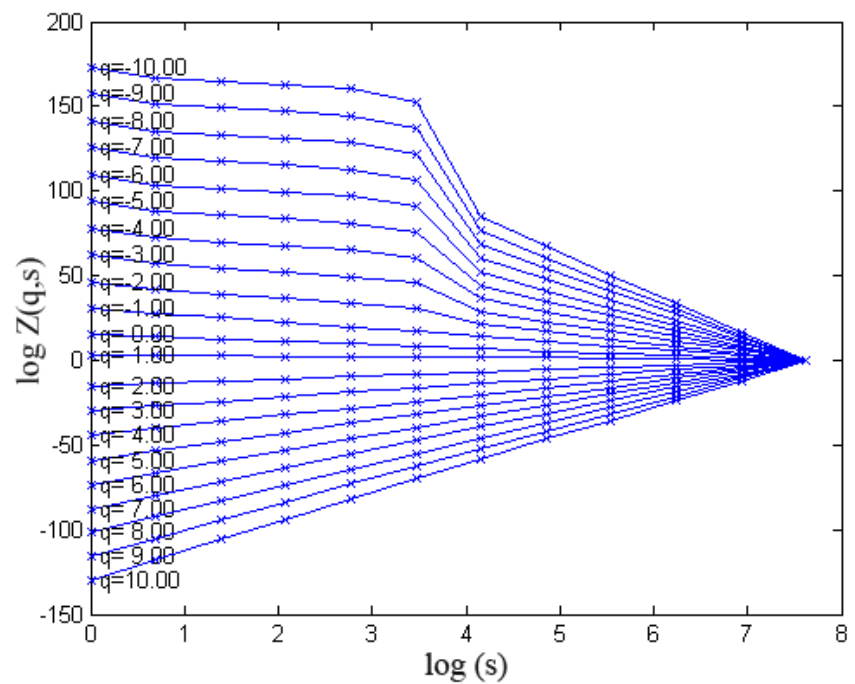

Fig. 18. Bi-log plot of partition function versus box-size for dye mass image.

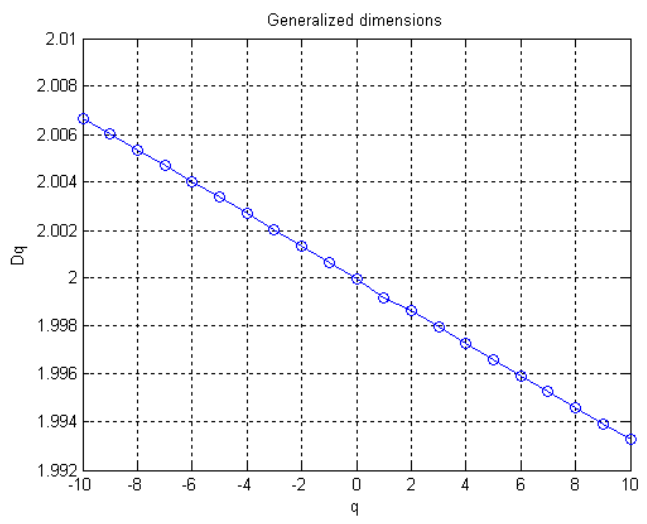

Fig. 19. Generalized fractal dimension for the dye mass image.

occurs only for scales larger than $s \geq 8$, so maxima lines revealing that scaling pattern do not propagate adequately.

However analysis of Lipschitz exponents along maxima lines is still convenient for our analysis providing important information about distribution of singularities (and so, the $f(\alpha)$ spectrum). As suggested in Zhong and Ning (2005) and Struzik (1999) it is possible to evaluate singular spectrum locally using Eq. (9) and tracing an histogram of the number of pixels within a certain interval of $\alpha$ values. First results for the dye mass image along the scales where a multifractal behaviour is expected are given in Fig. 20 where the centroid of the histogram equals $\alpha_{0}=1.77$, approximately equal to the computed value with box-counting method which equals $\alpha_{0}=1.83$.

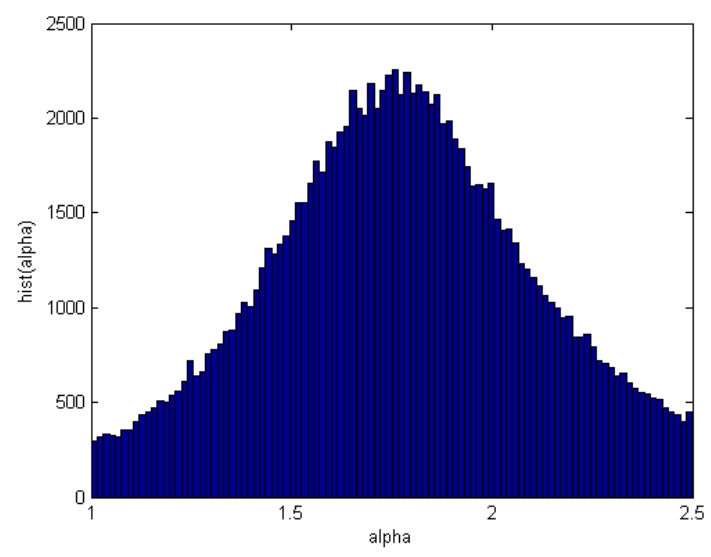

Fig. 20. Histogram of Local Lipschitz exponents for the dye mass image.

\section{Conclusions}

The application of the CWT (Continuos wavelet transform) and its particular representation called WTMM (Wavelet transform modulus maxima) to multifractal analysis has almost reached the status of a standard in natural phenomena analysis contributing to substantial progress in each domain where it has been applied.

This paper reviews the main concepts involved in the multifractal formalism and its relation with the signal representation obtained using the wavelet transform. The selected domain of application has been Hydrology, where different authors relate the permeability of different materials to the multifractal spectrum.

Some experiments using a dye tracer over a clay soil has been done, mainly focusing on the multifractal spectrum of the dye mass and dye depth quantities. Previous results by some of the authors (Tarquis et al., 2006) related with other parameters such as configuration entropy are also revisited trying to provide a complete set of measures capable of characterize soil properties.

Classical multifractal characterization with box-counting methods are given both for each horizontal section and for the dye mass image which shows that only for larger scales a multifractal behavior is expected. This is the main reason behind unexpected results obtained with wavelet extension of methods exposed in Sect. 4. However, if we plot an histogram of coefficients $\alpha$ for selected scales we obtain a very good approximation of the multifractal spectrum with the advantage that we precisely know the location of different $\alpha$-Lipschitz exponents. This may be an important and complementary information.

Apart from that, the main focus of future research is to extend the analysis with larger number of image sets to verify the significance of the results including theoretical multifractal images such as, for example, Sierpinsky carpets generators (Perfect et al, 2006). 
Acknowledgements. This research has been supported by the National Spanish Research Institution "Comisión Interministerial de Ciencia y Tecnología-CICYT" as part of the project AGL2006-12689/AGR and by Autonomic Institutions "Comunidad Autónoma de Madrid - CAM" and Technical University of Madrid - UPM as part of the project R05/11261.

Edited by: Q. Cheng

Reviewed by: two anonymous referees

\section{References}

Arneodo, A., Grasseau, G., and Holshneider, M.: Wavelet transform of multifractals, Phys. Rev. Lett., 61, 2281-2284, 1988.

Arneodo, A., Bacry, E., and Muzy, J.: Solving the inverse fractal problem from wavelet analysis, Europhysics Lett., 25(7), 479484, 1994.

Daubechies, I.: Ten lectures on wavelets, volume 61, CBMS conference on wavelets, 1992.

Folorunso, O. A., Puente, D. E., Rolston, D. E., and Pinzon, J. E.: Statistical and fractal evaluation of the spatial characteristics of soil surface strength, Soil Sci. Soc. Am. J., 58, 284-295, 1994.

Hsung, T., Lun, D., and Siu, W.: Denoising by singularity detection, IEEE Trans. Signal Processing, 47, 3139-3144, 1999.

Kravchenko, A. N., Boast, C. W., and Bullock, D. G.: Multifractal analysis of soil spatial variability, Agronomy J., 91, 1033-1041, 1999.
Mallat, S.: A Wavelet Tour of Signal Processing, Academic Press, 2nd edition, 1999.

Mallat, S.: A theory for multiresolution signal decomposition: The wavelet representation. IEEE Trans. Pattern, Recognition and Machine Intelligence, 11(7), 674-693, 1989.

Muller, J.: Characterization of pore space in chalk by multifractal analysis, J. Hydrol., 187, 215-222, 1996.

Pachepsky, Y. A., Giménez, D., Crawford, J. W., and Rawls, W. J.: Conventional and fractal geometry in soil science, in: Fractals in Soil Science, edited by: Pachepsky, Y. A., Crawford, J. W., and Rawls, W. J., Elsevier, Amsterdam, 2000.

Perfect, E., Gentry, W., Sukop, M. C., and Lawson, J. E.: Multifractal Sierpinsky carpets: Theory and application to upscaling effective saturated hydraulic conductivity, Geoderma, 134, 240252, 2006.

Struzik, Z. R.: Direct multifractal spectrum calculation from the wavelet transform. Technical Report INS-R9914, Information Systems, ISSN: 1386-3681, Amsterdam, 1999.

Tarquis, A. M., McInnes, K., Keys, J., Saa, A., Garcia, M. R., and Diaz, M. C.: Multiscaling analysis in a structured clay soil using 2D images, J. Hydrol., 322, 236-246, 2006.

Zhong, J. and Ning, R.: Image denoising based on wavelets and multifractals for singularity detection, IEEE Trans. on Image Processing, 14, 1435-1447, 2005. 